\title{
Obamacare and a Fix for the IRS Iteration Samuel J. Ferguson
}

\section{Introduction}

In 2018, I took an Uber ride. Although my driver qualified for help with paying for health insurance under the Affordable Care Act, he couldn't determine the amount of his benefit. Worse, tax software and government calculators said he should receive $\$ 0$ to help him pay for health insurance, instead of the roughly $\$ 3,000$ that the law seemed to prescribe. He asked me to look into the matter, and my development of a bisection algorithm led to a mathematical odyssey captured by a senior writer at Money and Time's film crew in an article [6] and film clip [7]. After communication with the IRS [12], I was motivated to publish this paper in a peer-reviewed publication so that the IRS can reference it in their tax guidance. Then, tax software will be able to implement procedures proposed here without legal liability, relieving the current computational issues affecting beneficiaries. I am also motivated by the opportunity to bear witness to the resolution of a civic concern by means of modeling and proof.

\section{Obamacare's Premium Tax Credit}

A tax device created by the Affordable Care Act [9], also called Obamacare, plays a key role in my driver's problem. Passed in 2010, Obamacare establishes online exchanges from which American households can directly purchase health insurance meeting certain standards. The law also makes health insurance affordable ${ }^{1}$ for every American

Samuel J. Ferguson is a research scientist at Metron, Inc. His email address is ferguson@metsci .com.

${ }^{1}$ We postpone defining "affordable" and "household income." The latter is the sum of the household members' modified adjusted gross incomes (MAGI), hence our use of the letter " $M$ " to denote it. Worksheets for calculating $M$ may be found in the Instructions for Form 8962 [15, p. 6].

Communicated by Notices Associate Editor Reza Malek-Madani.

For permission to reprint this article, please contact:

reprint-permission@ams .org. household with

$$
M=\text { household income }
$$

in the range

$$
F \leq M \leq 4 F,
$$

where

$$
F=\text { federal poverty line. }
$$

Here, the federal poverty $\operatorname{line}^{2}$ for the household is a governmentally-prescribed number depending on household size and state which adjusts annually.

How does Obamacare make health insurance affordable when $M$ is in $[F, 4 F]$ ? It creates a tax credit to help eligible households pay the premiums of qualified insurance. For such households, and some health plans from exchanges, the credit pays all of the premiums except a portion which is considered affordable. How much of $M$ is that? The applicable figure $f$ represents the percentage of $M$ which is affordable for the household to pay for premiums, and is obtained in Form 8962 [13], which must be filed to claim the tax credit. We model $f$ as a function of

$$
m=M / F \text {. }
$$

Example. The applicable figure $f(m)$ for 2018 is modeled ${ }^{3}$

\footnotetext{
${ }^{2}$ The value of $F$ used in Obamacare calculations for a given tax year may be found in the Instructions for Form 8962 for that year [15, pp. 6-7]. For example, in the continental United States in 2018, for a household with $n$ people, $F$ is approximately $8,000+n \cdot 4,000[14$, p. 7] dollars.

${ }^{3}$ The values $j=f(1), k=f(1.33), \ell=f(1.5), a=f(2), b=f(2.5)$, $c=f(3)$ are from Table 2 in the Instructions for Form 8962 for 2018 [14, p. 9]. Given $n \%$ in the right column of Table 2, rounding $f\left(\frac{n}{100}\right)$ to the nearest tenthousandth yields the corresponding decimal on the left column. To model the applicable figures for 2019, say, we use the same functional form with the values $j=0.0208, k=0.0311, \ell=0.0415, a=0.0654, b=0.0836, c=0.0986$ instead [15, p. 9].
} 


\section{COMMUNICATION}

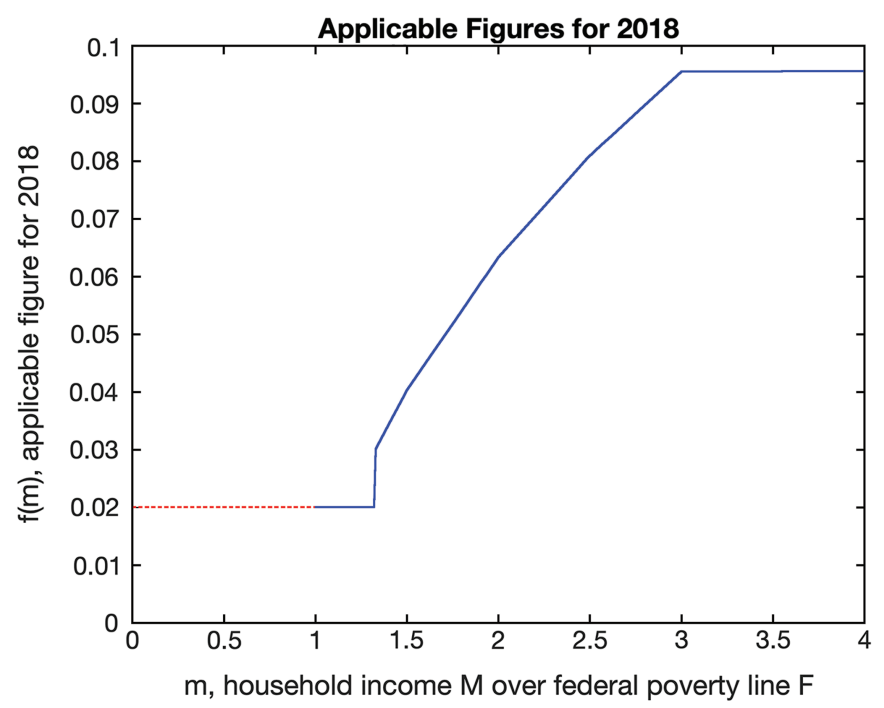

Figure 1. A graph of the applicable figure $f(m)$ as a function of $m=M / F$. The solid graph is for $m$ in $[1,4]$. For $m<1$, the value of the dashed line is $f(1)$, used for eligible households. No applicable figure is needed when $m>4$.

by defining $f(m)$ as

$$
f(m)= \begin{cases}j, & 0 \leq m<1.33, \\ k+(\ell-k) \frac{m-1.33}{1.5-1.33}, & 1.33 \leq m<1.5, \\ \ell+(a-\ell) \frac{m-1.5}{2-1.5}, & 1.5 \leq m<2, \\ a+(b-a) \frac{m-2}{2.5-2}, & 2 \leq m<2.5, \\ b+(c-b) \frac{m-2.5}{3-2.5}, & 2.5 \leq m<3, \\ c, & 3 \leq m \leq 4,\end{cases}
$$

where $(j, k, \ell, a, b, c)=(0.0201,0.0302,0.0403,0.0634$, $0.0810,0.0956)$. See Figure 1 . From the definition, we see that the applicable figure is nondecreasing, discontinuous, and right continuous, as a function of $m$.

In general, if $M$ is in $[F, 4 F]$, then $m=M / F$ is in $[1,4]$ and $f(m)$ is defined. If $m>4$, then household income is too high to receive the premium tax credit, so no applicable figure is needed. If $m<1$, then household income is too low, unless the household qualifies for an exception $[15$, p. 8], in which case the value of the applicable figure is $f(1)$. As in 2018, the model for the applicable figure is always nondecreasing and right continuous as a function of $m$ for all tax years.

Now, how do we compute the amount of the premium tax credit (PTC)? First, we need to know the costs of premiums. There may be many health plans on the exchange, but one will serve as a benchmark for computing the PTC, with a cost of

$$
P=\text { annual benchmark premium. }
$$

The qualified plans actually purchased for the household members may differ from the household's benchmark plan, and the sum of their costs is

$$
Q=\text { annual enrollment premiums. }
$$

If the benchmark plan is actually purchased, then the amount of the PTC is generally all of $P$ except for the portion of income which is considered affordable to pay, the annual contribution amount $f(m) \cdot M$. Thus, the PTC is $\max (0, P-f(m) \cdot M)$ in this case, since a tax credit must be nonnegative. In general, if we allow any qualified plans, the PTC cannot exceed $Q$, the cost of insurance actually purchased. This gives the general expression for the PTC as a function of $M$ as

$\operatorname{PTC}(M)= \begin{cases}\min (Q, \max (0, P-f(m) \cdot M)), & 0 \leq M \leq 4 F, \\ 0, & M>4 F,\end{cases}$

if the household is eligible except for possibly having an income that is too high.

Simplified Example. Suppose we have a household of one person in 2018. Assume for simplicity that $M=50,000$, $F=12,500$, and the benchmark premium is 500 dollars per month for the household. Then, the annual benchmark premium ${ }^{4}$ is

$$
P=12 \cdot 500=6,000
$$

dollars. As $f(4)=0.0956$, the annual contribution amount is

$$
f(m) \cdot M=0.0956 \cdot 50,000=4,780
$$

dollars. If the benchmark insurance is actually purchased, so $Q=P$, then the government pays the rest, which is

$$
P-f(m) \cdot M=6,000-4,780=1,220
$$

dollars. Thus, $\operatorname{PTC}(M)=1,220$ and $\$ 1,220$ is the amount of Obamacare's premium tax credit for the household. See Figure 2 for a plot of PTC $(M)$ as $M$ varies. As Figure 2 suggests, the premium tax credit is decreasing as a function of $M$ and right continuous on $[0,4 F]$. It typically has discontinuities at $(1.33) F$ and $4 F$.

Enrollees receive a form ${ }^{5}$ giving the values they should use for $Q$ and $P$ when filing taxes. To recap, the instructions for claiming the premium tax credit give the values of $F$

\footnotetext{
${ }^{4}$ More precisely, $P$ is the sum of the unsubsidized or "sticker price" annual benchmark premiums for the household members. The benchmark plan for each individual is the "second lowest cost silver plan" (SLCSP) on the exchange for them. The prices for an individual may depend on their age and smoking habits in addition to their county and the year. If enrollees, plans, or premiums change monthly, then each month's premiums must be recorded. And, in certain circumstances, costs are split between multiple tax returns. We assume, for simplicity, that neither of these occur.

${ }^{5}$ Form 1095-A lists annual values for $Q$ and $P$ on lines $33 A$ and $33 B$ for each household [13]. It also gives the annual value of advance payments of premium tax credits (APTC) on line 33C.
} 


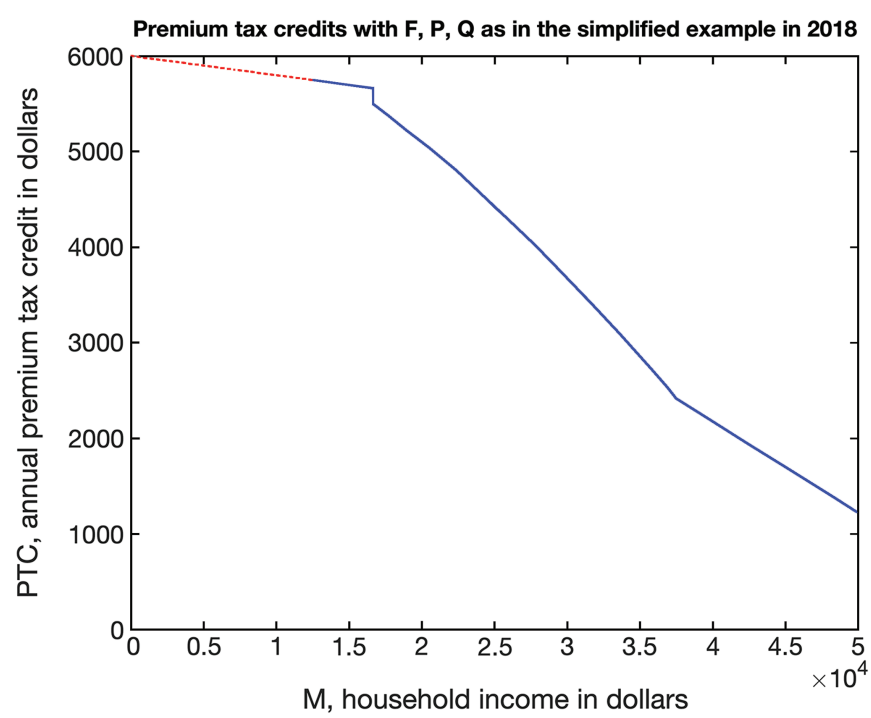

Figure 2. A graph of the tax credit PTC $(M)$ as a function of household income $M$, where $F=12,500, P=6,000$, and $Q=P$, as in the simplified example. The applicable figures are those of Figure 1. For $M<F$, the dashed line is PTC if there is an exception to ineligibility. The premium tax credit is 0 if $M>4 F$.

and, once $M$ is known, $f(m)$ from tables. Then, using $Q, P$ read off a form, the premium tax credit is calculated by the expression above. With such a precise process available, why was my driver unable to find his premium tax credit?

\section{The Circular Relationship}

We have yet to examine household income $M$. For households with income from self-employment-an independent contractor, a private tutor, and a driver on a ridesharing app are all likely self-employed-the value of $M$ can be tricky to find for households eligible for the PTC. In 2014, self-employed workers were "almost three times more likely" than others to obtain health insurance from the Obamacare exchanges, according to the Treasury [4], so self-employed households form a sizable proportion of beneficiary households.

Pinning down household income $M$ for self-employed households may be difficult because it involves a tax deduction $d$ which cannot exceed their out-of-pocket health insurance costs. For households also eligible for the PTC, the total cost $Q$ of health insurance is being shared with the government, which is contributing $\operatorname{PTC}(M)$. Thus, what the household pays out-of-pocket should be $Q-\operatorname{PTC}(M)$, and hence

$$
d \leq Q-\operatorname{PTC}(M) .
$$

We now come to my driver's dilemma. To find his premium tax credit $P T C(M)$, he must know $M$, which depends on $d$. As $d \leq Q-P T C(M)$, he must know his PTC to find out how large $d$ can be. But the PTC is what he wanted to find in the first place! So, there is a "circular relationship" in the United States tax code between the premium tax credit and the self-employed health insurance deduction [18]. This means that the Internal Revenue Service (IRS) has the following problem.

Problem 3.1. What is a procedure, computable by hand in a reasonable time, that finds the appropriate health insurance deduction $d$ for any self-employed household eligible for Obamacare's premium tax credit?

The "appropriate" choice of $d$ is the value maximizing the household's tax benefit, which is the sum of the tax credit $\operatorname{PTC}(M)$ and the taxes saved by reducing income by $d$. If the tax function $T(\cdot)$ assigns, to a given income, the federal income tax before tax credits, then the taxes saved by reducing income $I$ by $d$ is $T(I)-T(I-d)$. The tax benefit is thus PTC $(M)+(T(I)-T(I-d))$. Hence, as $I$ is independent of $d$, the appropriate $d$ optimizes $\max _{d \leq Q-\operatorname{PTC}(M)} \operatorname{PTC}(M)-T(I-D)$. Essentially, we want to maximize the tax credit while minimizing the tax. It turns out that the bigger $d$ is, the smaller $M$ is, whence the bigger $\operatorname{PTC}(M)-T(I-d)$ is. Thus, the largest value of the latter occurs for the largest $d$ such that $d \leq Q-\operatorname{PTC}(M)$. That is, the appropriate nonnegative value of $d$ can be written as

$$
\max (\{d: d \leq Q-\operatorname{PTC}(M)\})
$$

if the household is eligible for the premium tax credit.

What does "computable by hand in a reasonable time" mean, above? Practically, it means the IRS can put it into tax guidance, meaning the IRS does not consider it overly onerous to require of a typical taxpayer with access only to its instructions and the ability to do arithmetic. For example, if we try all possible dollar values for $d$ satisfying the constraint, some value will yield the maximum tax benefit, and hence be appropriate. But the IRS likely considers trying every possible value to be a burdensome computational task for a typical American. Thus, our task is to create a dependable, reasonably fast procedure, involving just addition, subtraction, multiplication, division, and rounding, that works for all tax years. We turn now to current IRS guidance for self-employed taxpayers who qualify for the premium tax credit. This guidance can be viewed as an attempted solution of our problem.

\section{The IRS Fixed Point Iteration}

Current IRS guidance offers self-employed Obamacare beneficiaries two methods for determining allowable values of their self-employed health insurance deduction $d$ [17, pp. 62-65]. The second, "simplified calculation method," is a truncation of the first, "iterative calculation method," so we focus primarily on motivating and analyzing the IRS iterative method here. 
To motivate the IRS iteration for finding the deduction $d$, we ask what equations $d$ might satisfy. If we write the PTC as a variable, ptc, then $p t c=\operatorname{PTC}(M)$. Taking $M=M(u)$ to be a decreasing, continuous function of the deduction $u$, and defining

$$
\operatorname{PTC}(u)=\operatorname{PTC}(M(u))
$$

with a modest abuse of notation, we have ptc $=P T C(d)$. In addition, the appropriate $d$, satisfying the constraint $d \leq$ $Q-P T C(d)$, might attain the equality $d=Q-P T C(d)$. This means each dollar paid for health insurance leads to a dollar of tax credit or deductions, a plausible property for the $d$ maximizing the tax benefit. We arrive at a system of two equations in two unknowns given by

$$
(p t c, d)=(P T C(d), Q-P T C(d)) .
$$

In mathematical language, and in simple scenarios, the IRS iterative method begins with the point $\left(p t c_{1}, d_{1}\right)=$ $(0, Q)$ and, for $n \geq 1$, defines $\left(p t c_{n+1}, d_{n+1}\right)$ by

$$
\left(p t c_{n+1}, d_{n+1}\right)=\left(\operatorname{PTC}\left(d_{n}\right), Q-\operatorname{PTC}\left(d_{n}\right)\right) .
$$

If $\left(p t c_{n}, d_{n}\right) \rightarrow(p t c, d)$ as $n \rightarrow \infty$, then the left continuity of $P T C(d)$, inherited from the right continuity of $f(m)$, can be used to prove ${ }^{6}(p t c, d)=(P T C(d), Q-P T C(d))$, as desired. In this case, $d \leq Q-P T C(d)$ holds and, as $d+P T C(d)$ is a strictly increasing function of $d$, no larger value satisfies the inequality. Thus, $\lim _{n \rightarrow \infty} d_{n}$ is $\max (\{d: d \leq Q-P T C(d)\})$, the appropriate value, when the iteration converges.

We observe that if we define $G$ on an appropriate subset of $\mathbb{R}^{2}$ by

$$
G(x, y)=(P T C(y), Q-P T C(y)),
$$

then the equation defining the iteration becomes $X_{n+1}=$ $G\left(X_{n}\right)$ if $X_{n}=\left(p t c_{n}, d_{n}\right)$ for $n \geq 1$. If $X_{n} \rightarrow X$ as $n \rightarrow \infty$, then $X=G(X)$, so $X$ is a point held fixed by $G$. As the IRS method seeks a fixed point, we may refer to it as a fixed point iteration.

Having motivated the IRS fixed point iteration with the usual notion of convergence, we point out that the IRS uses its own test for convergence, which we rephrase mathematically. Let us say the sequence $\left\{\left(p t c_{n}, d_{n}\right)\right\}_{n=1}^{\infty}$ converges in the IRS sense if, when $\left(p t c_{n+1}, d_{n+1}\right)$ is taken to be the result of rounding the entries of $G\left(p t c_{n}, d_{n}\right)$ to the nearest penny, there exists a positive integer $N$ such that

$$
\left\|\left(p t c_{k}, d_{k}\right)-\left(p t c_{j}, d_{j}\right)\right\|_{\infty}<\varepsilon_{0}
$$

for all integers $k, j \geq N$, with $\varepsilon_{0}=1$. The above norm is defined by $\|(x, y)\|_{\infty}=\max (|x|,|y|)$. The "iterative

\footnotetext{
${ }^{6}$ It is helpful to note that $d_{2} \leq d_{4} \leq d_{6} \leq \cdots$ when $\left(p t c_{1}, d_{1}\right)=(0, Q)$. Applying the left continuity of PTC $(\cdot)$ to the sequence $\left\{d_{2 n}\right\}_{n=1}^{\infty}$ yields $P T C(d)=$ $\lim _{n \rightarrow \infty} \operatorname{PTC}\left(d_{2 n}\right)=$ ptc.
}

calculation method" amounts to taking $d$ to be the $y$ coordinate of $\left(p t c_{n_{0}}, d_{n_{0}}\right)$ after rounding, where $n_{0}$ is the smallest value of $N$ that satisfies our IRS convergence condition [17, p. 63]. The actual text of IRS guidance asks taxpayers to "not use the iterative calculation method" if $\left\|\left(p t c_{n+1}, d_{n+1}\right)-\left(p t c_{n}, d_{n}\right)\right\|_{\infty} \geq \varepsilon_{0}$ for all $n \geq 1$. The oscillatory nature of the sequences implies that this holds if and only if $\left\{\left(p t c_{n}, d_{n}\right)\right\}_{n=1}^{\infty}$ fails to converge in the IRS sense $[17$, p. 64]. Thus, for sequences coming from its fixed point iteration, the IRS provides a simple, consistent test ${ }^{7}$ for divergence and convergence.

IRS guidance also offers its simplified calculation method, which amounts to asking beneficiaries to take $d_{2}$ as their health insurance deduction and, hence, $p t c_{3}$ as their premium tax credit. When $\left\{\left(p t c_{n}, d_{n}\right)\right\}_{n=1}^{\infty}$ fails to converge in the IRS sense, these are the values that IRS guidance currently arrives at for $d$ and ptc. The best tax software may extend the simplified procedure, so taxpayers take at most

$$
d_{0}=\liminf _{n \rightarrow \infty} d_{n}
$$

as their deduction, and hence PTC $\left(d_{0}\right)$ as their premium tax credit. When we don't have convergence in the IRS sense, however, $d_{0}$ is generally smaller than the appropriate value, and in many cases $P T C\left(d_{0}\right)$ yields a premium tax credit of $\$ 0$. This is apparently the cause of my driver's difficulty, and we emphasize that these inappropriate values are what tax software and government calculators give now.

Unsurprisingly, the IRS says that self-employed taxpayers "may have difficulty" computing their premium tax credit, according to the IRS document which introduced the fixed point iteration [18]. However, IRS guidance says that "any" computation method may be used to find the appropriate deduction, provided it respects the constraint on $d$ and the separate rules for the deduction and credit $[17$, p. 64]. The below example proves that neither IRS method always works to compute appropriate values, so the fact that any valid method may be used gives us a fresh opportunity to solve the IRS problem-and my driver's dilemma.

Example. Consider the 2018 tax year, so we can use the applicable figure function $f(m)$ given previously. Suppose $I \geq Q$ to avoid tax complications, and let

$$
M(u)=I-u .
$$

Here, $I$ is earned income from self-employment in a single

\footnotetext{
${ }^{7}$ This divergence test is equivalent to using the well-known Cauchy criterion with $\varepsilon_{0}=1$ dollar.
} 
business. Then

$$
\begin{aligned}
& \operatorname{PTC}(u) \\
& = \begin{cases}\min \left(Q, \max \left(0, P-f\left(\frac{I-u}{F}\right) \cdot(I-u)\right)\right), & 0 \leq \frac{I-u}{F} \leq 4, \\
0, & \frac{I-u}{F}>4 .\end{cases}
\end{aligned}
$$

Say we have a household of two in Brooklyn, New York, consisting of an individual and a dependent child who is less than 26 years old. The household's federal poverty line is $F=16,240$ [14, p. 7]. Data for the county, Kings County, show that the cost of benchmark premiums for the household is $\$ 865.81$ per month or, to the nearest dollar, $P=10,390$ annually [5]. Suppose that the household has earned self-employment income from a single business of $I=71,150$, and take $Q=P$. Following the IRS iteration, and rounding to the nearest dollar in intermediate steps for simplicity, we obtain $\left(p t c_{1}, d_{1}\right)=(0,10,390)$ and

$$
p t c_{2}=10,390-0.0956 \cdot 60,760,
$$

as $71,150-10,390=60,760$. Hence, after rounding, $p t c_{2}$ is 4,581 . Thus,

$$
d_{2}=10,390-4,581=5,809 .
$$

In turn, this makes $I-d_{2}=65,341$, which is greater than $4 F=64,960$. So, by definition, $p t c_{3}=0$. Yet this yields $d_{3}=10,390$, putting us back where we started. Hence, the sequence doesn't converge in the IRS sense. On the other hand, if we follow the simplified calculation method, we arrive at a deduction of $d_{2}=5,809$ and a premium tax credit of $p t c_{3}=0$. This is worse than not claiming the premium tax credit at all, and letting $d=10,390$. It turns out that the 0 value for the premium tax credit is not appropriate, as we shall see. If we progressively narrow our search for the deduction by performing repeated bisections, say, then we can do better.

\section{Bisection Methods}

We now propose bisection procedures for finding the appropriate self-employed health insurance deduction $d$. We first adapt the well-known bisection proof of the Intermediate Value Theorem to left continuous, monotone increasing functions.

Theorem 5.1. Let $g$ be a real-valued, increasing, left continuous function on an interval $[a, b]$, and let $k$ be a given real number. If $g(a) \leq k$, then there exists a real number $d$ in $[a, b]$ such that $g(d) \leq k$ and $g(u)>k$ whenever $u>d$ in $[a, b]$.

Proof. If $g(b) \leq k$, then, as no $u>b$ exists in $[a, b]$, set $d=b$. Otherwise, if $g(b)>k$, denote the midpoint of $[a, b]$ by $d_{1}=\frac{a+b}{2}$. If $g\left(d_{1}\right)>k$, set $a_{1}=a, b_{1}=d_{1}$, and reduce our search to $\left[a_{1}, b_{1}\right]$. If $g\left(d_{1}\right) \leq k$, set $a_{1}=d_{1}$, $b_{1}=b$, and again reduce our search to $\left[a_{1}, b_{1}\right]$. Repeated bisection leads to the largest $d$ in $[a, b]$ such that $g(d) \leq k$. The details are in a version of this article with a complete proof [3].

The $d$ found by bisection in the proof satisfies $g(d) \leq k$ due to the left continuity of $g$, and is the largest $d$ with this property because $g$ is increasing. We can adapt this theorem and proof to justify using bisection to calculate the self-employed health insurance deduction $d$ as in the two procedures of the following corollary.

Corollary 5.2. Suppose that $F, P, Q>0, f:[0,4] \rightarrow$ $(0,1)$ is a right continuous, nondecreasing function, $M$ : $[0, Q] \rightarrow[0, \infty)$ is a continuous, decreasing function, and PTC $:[0, \infty) \rightarrow[0, Q]$ is given by

$$
\begin{aligned}
& \operatorname{PTC}(M) \\
& = \begin{cases}\min \left(Q, \max \left(0, P-f\left(\frac{M}{F}\right) \cdot M\right)\right), & 0 \leq M \leq 4 F, \\
0, & M>4 F .\end{cases}
\end{aligned}
$$

Let PTC : $[0, Q] \rightarrow[0, Q]$ be given by PTC $(u)=\operatorname{PTC}(M(u))$. When restricted to $J=\{u \in[0, Q]: 0 \leq M(u) \leq 4 F\}, P T C$ is increasing and left continuous. If $J$ is nonempty, so we can write $[a, b]=J$, and $g:[a, b] \rightarrow[0, \infty)$ given by $g(u)=$ $u+P T C(u)$ satisfies $g(a) \leq Q$, then bisection yields the largest $d$ in $[a, b]$ such that $g(d) \leq Q$. Moreover, if $g(u)=u+P T C(u)$ is defined on $[0, Q]$, bisection yields the largest $d$ in $[0, Q]$ such that $g(d) \leq Q$ if the value d satisfies $M(d) \neq 4 F$.

These two bisection methods apply because " $g(d) \leq Q$ " is equivalent to $d$ satisfying $d \leq Q-P T C(d)$. For a selfemployed household, eligibility for the PTC implies $g(u) \leq$ $Q$ and $P T C(u)>0$ for some $u \in[0, Q]$, and the latter implies $M(u) \leq 4 F$, so the hypotheses of the first method are met. The second method works because PTC $(M)$ is right continuous in $M$ except perhaps at $M=4 F$.

Example. We can perform the second bisection method on the example from the preceding section. After thirteen bisections of the interval $[0, Q]$, rounding to the nearest dollar after each step for simplicity, we find that the appropriate deduction is $d=6,208$. From this, we see that the appropriate premium tax credit for the household is $\operatorname{PTC}(d)=4,182$, or $\$ 4,182$, substantially more than the $\$ 0$ arrived at by the simplified calculation method. Given $d=6,208$, and without performing bisection, it is readily checked that this value cannot be improved because $d+4,182=Q$ and $u \mapsto u+P T C(u)$ is strictly increasing. By means of such checking, the IRS apparently verifies the correctness of tax returns prepared using values of $d$ found by methods outside of its guidance.

Similarly to the above example, when $Q=P, M(u)=$ $I-u$, and $Q \geq f(4) \cdot 4 F$, there is generally an interval of 
incomes $I \leq 4 F+f(4) \cdot 4 F$ for which the IRS fixed point iteration breaks down. In this case, the simplified calculation method gives $\$ 0$ for the PTC, yet an appropriate deduction $d$ can be found by bisection yielding substantial premium tax credits, often worth thousands. For a practical explanation of how to override the self-employed health insurance deduction and premium tax credit computations in tax software with appropriate values obtained by bisection, see the recent post [10] on the blog The Finance Buff.

Bisection also offers improvement over IRS guidance near $m=1.33$, as the discontinuity in $f(m)$ at 1.33 again prevents IRS convergence nearby. In fact, it is possible for the equation $d+P T C(d)=Q$ to have no solution for some interval of incomes $I$, due to the discontinuity at $m=1.33$. For such $I$, there is a value $d$ such that $d+P T C(d)<Q$ yet $u+P T C(u)>Q$ for $u>d$ due to a discontinuous jump in PTC $(u)$ as $u$ approaches $d$ from the right. Thus, this $d$ is the appropriate value for the deduction, yet $d+P T C(d)<Q$. In our model of the IRS fixed point iteration, if $\left\{\left(p t c_{n}, d_{n}\right)\right\}_{n=1}^{\infty}$ converges to $(p t c, d)$, then $d+P T C(d)=Q$ necessarily follows. For this reason, in this interval of incomes $I$, it is impossible for the IRS iteration to converge, no matter how we select the initial point $\left(p t c_{1}, d_{1}\right)$.

Example. Say that a household of one has earned income $I$ from self-employment of $\$ 16,300$, their federal poverty line $F$ is $\$ 12,000$, and they purchase the benchmark health plan from the exchange, with an annual cost $Q$ of $\$ 5,000$. Then, for a simple tax return using the applicable figures from 2018, the equation $d+P T C(d)=Q$ has no solution $d$ for the household. Indeed, if a deduction $d=341$ or higher is proposed, we arrive at a household income of $M=I-d=19,959$ or lower. This leads to $m<1.33$, so $f(m)=0.0201$, whence the household has a contribution amount of $0.0201 \cdot 19,959=321$ or lower. Thus, the premium tax credit is $5,000-321=4,679$ or higher, and so the sum of the deduction and credit is 5,020 dollars or higher, hence $d+P T C(d)>Q$. On the other hand, if a deduction $d=340$ or lower is proposed, we arrive at a household income of $M=I-d=19,960$ or higher. This leads to $m \geq 1.33$, so $f(m)$ is 0.0302 or higher, whence the household has a contribution amount of $0.0302 \cdot 19,960=482$ or higher. Thus, the premium tax credit is $5,000-482=4,518$ or lower, and so the sum of the deduction and credit is $\$ 4,858$ or lower, hence $d+P T C(d)<Q$. Thus, for all possible values of $d$, we have $d+P T C(d) \neq Q$. In other words, the equation $d+P T C(d)=Q$ has no solution $d$, and so the IRS iteration cannot converge in this case.

\section{Further Questions}

Having come this far with our bisection model, here we briefly indicate some directions in which additional complexities of the tax code can be taken into account, and also suggest further questions for future research.

The first direction in which we can expand our analysis is by examining the function $u \mapsto M(u)$ more closely in realistic applications, at least for the current configuration of the tax code. While $M(u)=I-u$ in our examples, where $I$ is earned income from self-employment in a single business, this need only be true for "simple" tax returns, defined in another version of this article [3]. For general tax returns, additional sources of income and most deductions merely cause translations in $u \mapsto M(u)$. Nevertheless, additional deductions can depend on $d$, and a short list of them is available [17, p. 65]. An example is the student loan interest deduction [16, p. 37]. Although these can increase the complexity of the appropriate function $u \mapsto M(u)$, each of them "phases out" in a continuous way as income increases, so these deductions are nondecreasing and continuous in $d$. An upshot of this is that in general tax returns, $u \mapsto M(u)$ can still be modeled as a continuous, decreasing function of $u$, and the applicability of bisection persists.

A second direction in which our analysis can be enhanced is by examining the effect of advance payments of the premium tax credit (APTC) on the calculation of $d$ and the total tax benefit. The first effect is that, as the APTC is paid directly to the insurance company in monthly installments, and the deduction $d$ cannot exceed the outof-pocket costs that the household incurred during the year, we have the additional constraint $d \leq Q-A P T C$, where APTC is the APTC's annual value. This can be taken into account by replacing $[0, Q]$ with $[0, Q-A P T C]$ in the above corollary. The second effect is that, when taxes are filed and the APTC must be reconciled with the appropriate PTC, the tax benefit can be different from what was used in our model. When $P T C(d) \geq A P T C$, the taxpayer receives an additional amount $P T C(d)-A P T C$ when filing taxes, giving a total of $P T C(d)$ in tax credits as before. When $\operatorname{PTC}(d)<A P T C$, however, the taxpayer only repays $\min \left(A P T C-P T C(d), R\left(\frac{M(d)}{F}\right)\right)$, where $R(m)$ is the repayment limitation, defined in another version of this article [3]. When the repayment limitation is less than $A P T C-P T C(d)$, the total tax benefit from Obamacare, $A P T C-R(m)$, exceeds $P T C(d)$. Nevertheless, the value of $d$ in $[0, Q-A P T C]$ found by the corollary is still optimal when taking into account the total tax benefit [3].

A third direction, for clarity rather than to account for tax complexities, is to improve our quantitative understanding of when the methods presented here can break down. For example, in applications, how big is the "typical" interval of incomes in which the IRS fixed point iteration breaks down near $f^{\prime}$ s discontinuity at $m=1.33$ ? 
As another example, what changes to the tax code would cause the bisection methods depicted here to fail? A new deduction that "phases in" in a discontinuous way might conceivably cause $u \mapsto M(u)$ to be neither continuous nor increasing, though this would be unintuitive. In turn, this could cause the bisection procedures indicated here to arrive at a deduction $d_{0}$ which fails to give the greatest tax benefit. While there are currently no deductions that phase in discontinuously, from 2005 to 2017 the domestic production activities deduction (DPAD) that might affect, say, someone who strikes oil in Texas, involved a continuous phase-in. Nevertheless, appropriate functions $u \mapsto M(u)$ were increasing in this period [3].

Additional questions might be concerned with policymaking, or the interplay between policy and tax guidance. For example, what are the simplest changes to Obamacare that would allow the IRS fixed point iteration to always converge to the correct value? If the tax credit for Obamacare phased out continuously as $M$ ranged above $400 \%$ of the federal poverty line, for example, this would eliminate the "subsidy cliff" discontinuity at $M=4 F$. If, further, Medicaid were expanded ${ }^{8}$ sufficiently to cover everyone near or below $f^{\prime}$ s discontinuity at $m=1.33$, this would appear to eliminate the other discontinuity, and the IRS iteration could be expected to always converge. In fact, as this article is going to press, recent coronavirus relief legislation is apparently causing the applicable figure parameters to be reset and continuously extending the premium tax credit for $M>4 F$ for 2021 and 2022. With these changes, the IRS iteration will always ${ }^{9}$ converge for 2021 and 2022 tax returns [11]. Nevertheless, the fact that a circular relationship is in the US tax code in the first place suggests that there are further appropriate ways to involve mathematicians in realistic future policy choices. We hope

\footnotetext{
${ }^{8}$ The Affordable Care Act allocates funding to help expand Medicaid, which gives health coverage for many low-income people, to households with incomes below $138 \%$ of the federal poverty line. However, the Medicaid program is run jointly by the federal government and the states. To ensure that states cooperate in the Medicaid expansion, Obamacare provides for Medicaid funding to be reduced for states which refuse to expand the program along the lines it prescribes. The United States Supreme Court ruled [8] that the latter is "coercive" to the states, and struck down the reduced funding provision in 2012. In states that have not expanded Medicaid sufficiently, a household may fall into a "Medicaid gap" and be ineligible for both Medicaid and the premium tax credit, which typically requires an expectation that $M \geq F$ for eligibility. Luckily, if the household expects $M \geq F$ and receives Obamacare benefits accordingly, yet $M<F$ at the end of the tax year, tax credit eligibility is generally retained and the applicable figure $f(1)$ may be used. The Medicaid gap affects those who expect $M<F$.

${ }^{9}$ For 2021 and 2022, the applicable figures will be 0 for $m<1.5$, a linear increase from 0 to 0.02 for $1.5 \leq m<2$, a linear increase from 0.02 to 0.04 for $2 \leq m<2.5$, a linear increase from 0.04 to 0.06 for $2.5 \leq m<3$, a linear increase from 0.06 to 0.085 for $3 \leq m<4$, and 0.085 for $m \geq 4$, according to one source [11]. With $f(m)$ now possessing continuity on $[0, \infty)$ and retaining modest slopes, convergence of the IRS iteration is guaranteed for 2021 and 2022.
}

this mathematical excursion, resolving the concern raised by my driver, inspires mathematicians to look for these and other ways they can use mathematics to help address citizens' concerns and, more generally, issues that affect the people they meet in daily life.

ACKNOWLEDGMENTS. I wish to thank the anonymous reviewers, my colleagues, and everyone mentioned in the Money article [6] for all their help. I want to especially thank an anonymous engineer for helping create the first Obamacare calculator for the selfemployed [1], Harry Sit for hosting a version updated at least through 2021 on his blog The Finance Buff [2], and Xiaona Zhou at Brooklyn's City Tech for incorporating repayment limitations in her calculator [19]. Feedback about the updated calculator should be addressed to Harry Sit at tfb@thefinancebuff.com and feedback about the calculator that accounts for repayment limitations should be addressed to Xiaona Zhou at Xiaona .Zhou@mai 1 . citytech. cuny. edu to be incorporated. Further testing and refinement of such calculators could improve accessibility and accuracy of results for selfemployed Obamacare beneficiaries.

\section{References}

[1] Anonymous and Samuel J. Ferguson, Self-Employed ACA Subsidy Calculator, 2018. (Retrieved March 20, 2021) https://cims.nyu.edu/ ferguson /Ca7cu1ator\%20SE\%20ACA.htm7

[2] Anonymous, Samuel J. Ferguson, and Harry Sit, SelfEmployed ACA Subsidy Calculator, 2021. (Retrieved February 26,2021 ) https://thefinancebuff.com/se1f -employed-aca-subsidy-calculator

[3] Samuel J. Ferguson, Obamacare and a fix for the IRS iteration, arXiv:2008.03355, 2020.https://arxiv.org/abs $/ 2008.03355$

[4] Adam Looney and Kathryn Martin, One in five 2014 marketplace consumers was a small business owner or selfemployed, Treasury Notes, U.S. Department of the Treasury, January 12, 2017. (Retrieved March 20, 2021) https:// www.treasury.qov/connect/bloq/Paqes/One-in -Five-2014-Marketplace-Consumers-was-a-Sma11 -Business-Owner-or-Self-Employed.aspx

[5] NY State of Health, The Official Health Plan Marketplace, 2018 Monthly Premiums for Second Lowest Cost Silver Plans (SLCSP) by Coverage Family Type, 2018, (Retrieved April 21, 2020) https://info.nystateofhea7th .ny.gov/sites/default/fi 1es/Eng1ish\%20 $-\% 202018 \% 20$ SLCSP\%20Chart.pdf

[6] Elizabeth O'Brien, How a 27-year-old math whiz (and his Uber driver) found a big flaw in the IRS tax code, Money, April 16, 2018. (Retrieved December 21, 2020) https://money . com/irs-tax-problem-obamacare-subsidy/ 


\section{COMMUNICATION}

[7] Elizabeth O'Brien, How a 27-year-old math whiz (and his Uber driver) found a big flaw in an IRS tax formula, Money, April 16, 2018. (Video retrieved December 16, 2020) https://twitter.com/money/status 7986008838452133890

[8] P. 15, slip op., National Federation of Independent Business v. Sebelius, U.S. Sup. Ct., June 28, 2012. (Retrieved March 20, 2021) https://www. supremecourt -gov/opinions/11pdt/11-393c3a2.pdt

[9] Patient Protection and Affordable Care Act, 42 U.S.C. Section 18001 et seq., 2010. (Retrieved March 20, 2021) https://www.gpo.gov/fdsys/pkg/PLAW -111pub7148/pdf/PLAW-111pub1148.pdf

[10] Harry Sit, When Turbotax and H\&R Block give self-employed wrong ACA subsidy, The Finance Buff, March 9, 2021. (Retrieved March 20, 2021) https://thefinancebuff .com/when-tax-software-gives-self-employed -wrong-aca-premium-subsidy.htm]

[11] Harry Sit, The Finance Buff, personal communication, March 19, 2021.

[12] Stephen J. Toomey, IRS Office of Chief Counsel, United States Treasury, personal communication, February 2018.

[13] U.S. Department of the Treasury, Internal Revenue Service, Form 8962, 2019. (Retrieved April 19, 2020) https://www.irs.gov/pub/irs-prior/f8962-2019.pdf

[14] U.S. Department of the Treasury, Internal Revenue Service, Instructions for Form 8962, December 3, 2018. (Retrieved April 19, 2020) https://www.irs.gov/pub /irs-prior/i8962--2018.pdf

[15] U.S. Department of the Treasury, Internal Revenue Service, Instructions for Form 8962, October 29, 2019. (Retrieved April 21, 2020) https://www.irs.gov/pub /irs-prior/i8962--2019.pdf

[16] U.S. Department of the Treasury, Internal Revenue Service, Publication 970: Tax Benefits for Education, January 17, 2020. (Retrieved April 21, 2020)https://www.irs .gov/pub/irs-prior/p970--2019.pdf

[17] U.S. Department of the Treasury, Internal Revenue Service, Publication 974: Premium Tax Credit (PTC), November 20, 2019. (Retrieved April 19, 2020) https://www .irs.gov/pub/irs-prior/p974--2019.pdf

[18] U.S. Department of the Treasury, Internal Revenue Service, Rev. Proc. 2014-41, 2014. (Retrieved April 16, 2018) https://www.irs.gov/pub/irs-drop/rp-14 $-41 . p d t$

[19] Xiaona Zhou, Self-Employed ACA Subsidy Calculator, March 4, 2020. (Retrieved April 30, 2020) https://xiaonazhou.shinyapps.io/Obamacare _Calculator_Version_3/

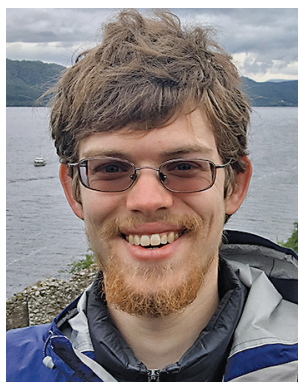

Samuel J. Ferguson

Credits

Figures are courtesy of Samuel J. Ferguson.

Photo of Samuel J. Ferguson is courtesy of Leland Ferguson.

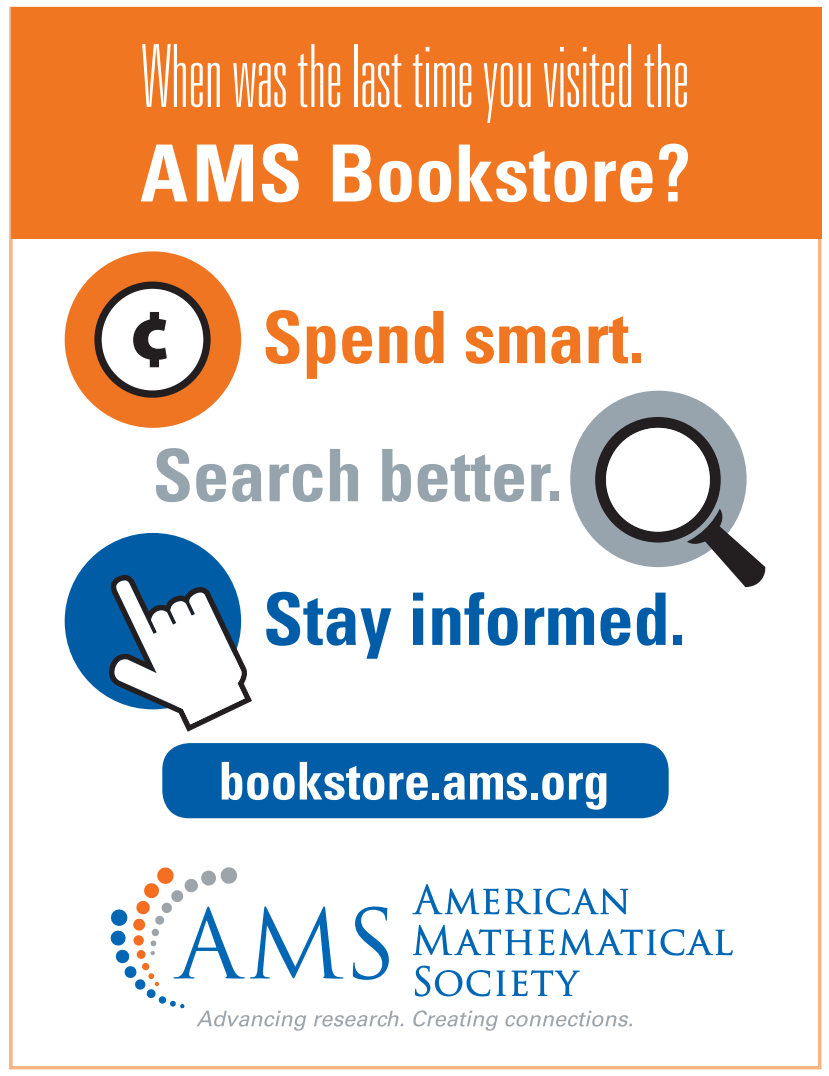

